\title{
Improved Sparse Channel Estimation for Cooperative Communication Systems
}

\author{
Guan Gui, ${ }^{1}$ Wei Peng, ${ }^{1}$ and Ling Wang ${ }^{2}$ \\ ${ }^{1}$ Department of Communication Engineering, Graduate School of Engineering, Tohoku University, Sendai 980-8579, Japan \\ ${ }^{2}$ School of Electronic Engineering, University of Electronic Science and Technology of China, Chengdu 610054, China
}

Correspondence should be addressed to Guan Gui, gui@mobile.ecei.tohoku.ac.jp

Received 30 May 2012; Revised 6 August 2012; Accepted 6 August 2012

Academic Editor: Sumei Sun

Copyright ( 2012 Guan Gui et al. This is an open access article distributed under the Creative Commons Attribution License, which permits unrestricted use, distribution, and reproduction in any medium, provided the original work is properly cited.

\begin{abstract}
Accurate channel state information (CSI) is necessary at receiver for coherent detection in amplify-and-forward (AF) cooperative communication systems. To estimate the channel, traditional methods, that is, least squares (LS) and least absolute shrinkage and selection operator (LASSO), are based on assumptions of either dense channel or global sparse channel. However, LS-based linear method neglects the inherent sparse structure information while LASSO-based sparse channel method cannot take full advantage of the prior information. Based on the partial sparse assumption of the cooperative channel model, we propose an improved channel estimation method with partial sparse constraint. At first, by using sparse decomposition theory, channel estimation is formulated as a compressive sensing problem. Secondly, the cooperative channel is reconstructed by LASSO with partial sparse constraint. Finally, numerical simulations are carried out to confirm the superiority of proposed methods over global sparse channel estimation methods.
\end{abstract}

\section{Introduction}

Relay-based cooperative communication [1-6] has been studied in the last decade due to its capability of enhancing the transmission range and providing the spatial diversity for single-antenna receivers by employing the relay nodes as virtual antennas [7-9]. A typical example of cooperative communication system is shown in Figure 1, where source $\mathbb{S}$ transmits signal to destination $\mathbb{D}$ with the help of relay $\mathbb{R}$. It is well known that utilizing multiple-inputs multiple-outputs (MIMO) transmission can boost the channel capacity [10, 11] in broadband communication systems. In addition, diversity techniques in MIMO system could mitigate selective fading and hence improve the quality of service (QoS) $[12,13]$. However, it poses a practical challenge to integrate multiple antennas onto a small handheld mobile terminal. To deal with the limitation, one could choose relay-based cooperation networks which have been investigated in last decade [1-5]. The main reason is that the diversity from relay nodes existing in the network could be exploited, where relay can either be provided by operators or be obtained from cooperating mobile terminals of other users.
In the relay-based cooperative communication system (CCS), data transmission is usually divided into two time slots. During the first time slot, the source broadcasts its information to both relay and destination. During the second time slot, the relay could select different protocols and then transmit the signal to the destination. Usually, there are two kinds of protocols in cooperative communication systems; one is to amplify the received signal at the relay and forward it to the destination, which is termed as amplifyand-forward (AF); the second is to decode the received signal, modulate it again, and then retransmit to destination, which is often termed as decode-and-forward (DF). Due to coherent detection in these systems, accurate channel state information (CSI) is required at the destination (for $\mathrm{AF}$ ) or at both relay and destination (for DF). About DF cooperative communication systems, the channel estimation methods could be borrowed from point-to-point (P2P) communication systems directly. However, extra channel estimation will increase the computational complexity which is a burden at relay, and broadcasting the estimated channel information will result in further interference at the destination. 


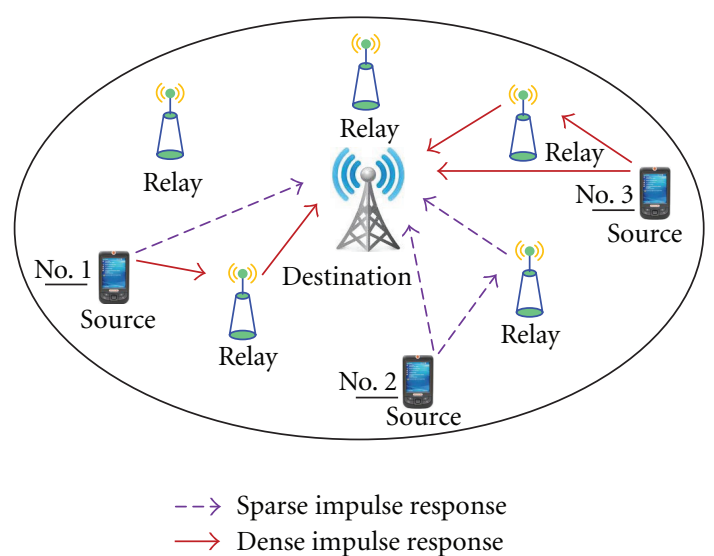

Figure 1: An example of AF broadband cooperative communication system, where source transmits signal to destination with the help of relay. No. 1, No. 2, and No. 3 are based on partial sparse, sparse, and dense channel model, respectively.

On the other hand, AF cooperative communication technique can avoid this disadvantage and we focus on AF CCS in this study.

As shown in Figure 1, the cooperative channel consists of a direct link $\mathbb{S} \Rightarrow \mathbb{D}$ and a cascaded one $\mathbb{S} \Rightarrow \mathbb{R} \Rightarrow \mathbb{D}$. Based on the assumption of dense multipath, linear channel estimation for the relay-based AF cooperative networks has been proposed [4]. Even though the proposed method can achieve lower-bound performance, low spectrum efficiency is unavoidable since the training sequence takes a large amount of the bandwidth. Hence, one method to improve the spectral efficiency is by reducing the length of training sequence for channel estimation.

As channel measurement techniques improved in the last decade, broadband wireless channels have been confirmed to exhibit inherent sparse or cluster-sparse structure in delay spread. If we can take advantage of the sparse prior information, then the spectral efficiency can be improved. In allusion to point-to-point (P2P) communication systems, efficient sparse channel estimation methods [14-16] have been proposed. To improve the spectral efficient and/or estimation performance in CCS, we have studied channel estimation in CCS and proposed an effective sparse channel estimation method [17].

However, the cascaded channel $(\mathbb{S} \Rightarrow \mathbb{R} \Rightarrow \mathbb{D})$ may be no longer sparse due to the linear convolutional operation [18]. Relay-based cooperative communication not only reduces the transmission range but also improves the received signal-to-noise ratio (SNR) when comparing with the P2P communication systems. Unlike the previous research under global sparse hypothesis, the cooperative channel consists of two parts: sparse part $(\mathbb{S} \Rightarrow \mathbb{D})$ and dense part $(\mathbb{S} \Rightarrow$ $\mathbb{R} \Rightarrow \mathbb{D})$. A simple example is shown in Figure 2. As a result, our previous method will be degraded since it cannot fully take advantage of the prior information. Unlike the previous method, in this paper, we propose a partial sparse channel estimation method by using LASSO [19] (PEL) to improve the performance. Based on this idea, an improved partial sparse channel estimation by using LASSO

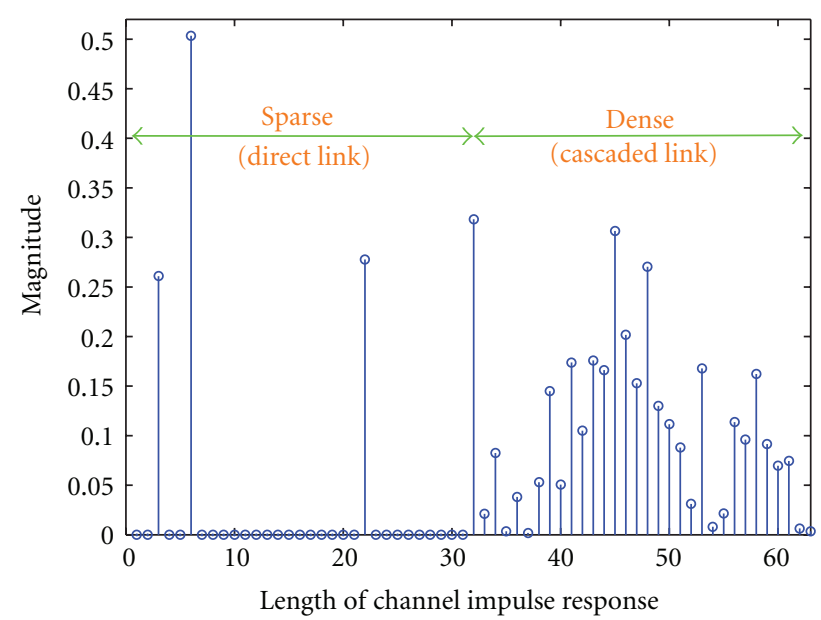

Figure 2: A typical example of partial sparse cooperative channel, where the first part of sparse impulse response is supported by direct link $(\mathbb{S} \Rightarrow \mathbb{D})$ and the second part of dense impulse response is contributed by the cascaded link $(\mathbb{S} \Rightarrow \mathbb{R} \Rightarrow \mathbb{D})$.

(IEL) is proposed by utilizing both partial sparse constraint and global sparse constraint. On the one hand, the partial sparse constraint can improve estimation performance in the cooperative communication systems. On the other hand, the global sparse constraint can mitigate noise interference under low SNR regime. To confirm the effectiveness of the two proposed methods, we give various numerical simulation results in Section 4.

Section 2 introduces the system model and problem formulation. In Section 3, two improved channel estimation methods are proposed. The first method is the improved channel estimation method by using partial sparse constraint, and the second one is an improved channel estimation method by using both partial and global sparse constraint. In Section 4, we give some representative numerical simulation results and related discussions. Concluding remarks are presented in Section 5.

Notations. In this paper, we use boldface lower case letters $\mathbf{x}$ to denote vectors and boldface capital letters $\mathbf{X}$ to denote matrices. $x$ represents the complex Gaussian random variable. $\mathbb{E}[\cdot]$ stands for the expectation operation. $\mathbf{X}^{T}, \mathbf{X}^{\dagger}$ denote transpose and conjugated transpose operations of $\mathbf{X}$, respectively. $\|\mathbf{x}\|_{0}$ is the number of nonzero elements of $\mathbf{x}$, and $\|\mathbf{x}\|_{2}$ is the Euclidean norm of $\mathbf{x}$. $\operatorname{diag}(\mathbf{x})$ represents a diagonal matrix whose diagonal entries are from vector $\mathbf{x}$. $\mathbf{x}_{1} * \mathbf{x}_{2}$ denotes the convolution of two vectors $\mathbf{x}_{1}$ and $\mathbf{x}_{2}$.

\section{System Model}

Consider a multipath fading AF CCS where the source $\mathbb{S}$ sends data to destination $\mathbb{D}$ with the help of relay $\mathbb{R}$ as shown in Figure 1. The three terminals are assumed to be equipped with a single antenna each. $\mathbf{h}_{S D}, \mathbf{h}_{S R}$, and $\mathbf{h}_{R D}$ denote the impulse response of the frequency selective fading channel vectors between three links $\mathbb{S} \Rightarrow \mathbb{D}, \mathbb{S} \Rightarrow \mathbb{R}$, and $\mathbb{R} \Rightarrow \mathbb{D}$, respectively. The three channel vectors are assumed to be 


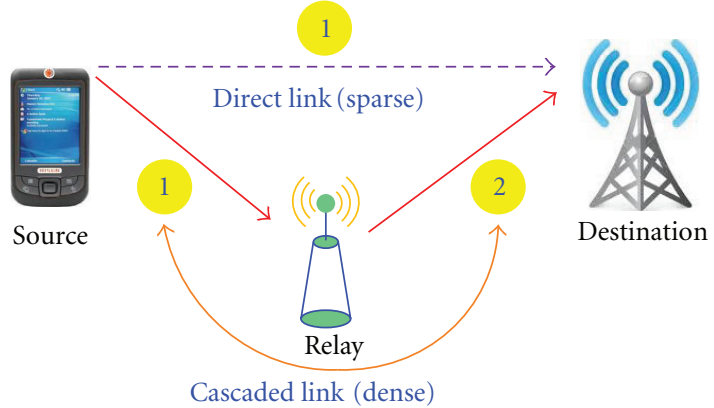

First time slot

Second time slot

Figure 3: Two-time slots partial sparse AF CCS.

zero-mean circularly symmetric complex Gaussian random variables with variance $\sigma_{h}^{2}$ and are independent of each other. For the time being, we assume perfect synchronization among three terminals. Note that they differ from our previous research in [17], because the impulse response of the cooperative channels, $\mathbf{h}_{S R}$ and $\mathbf{h}_{R D}$, is modeled as highly dense channel model due to the fact that the relay can reduce transmission range and improve channel quality. In other words, multipath taps arrive in a very short delay spread. The two channels are assumed to have length $L_{S R}$ and $L_{R D}$, respectively. For simplicity, we assume that they have same length $L_{S R}=L_{S R}=L / 2$, and the channel model of $\mathbf{h}_{S R}$ can be written as

$$
\mathbf{h}_{S R}=\sum_{l=0}^{L / 2-1} h_{S R, l} \delta\left(t-\tau_{S R, l}\right),
$$

where $h_{S R, l}$ and $\tau_{S R, l}$ represent the complex path gain with $\mathbb{E}\left[\sum_{l}\left|h_{S R, l}\right|^{2}\right]=1$ and symbol spaced time delay of the $l$ th path, respectively. The training sequence vector $\mathbf{x}$ is denoted as $\mathbf{x}=[x(1), x(2), \ldots, x(N)]^{T}$ where $N$ is the number of training length, and the transmit power is $P_{S}=\mathbb{E}\left[\mathbf{x}^{H} \mathbf{x}\right]=$ $N P$, where $P$ is the symbol power. According to the property of AF cooperative system, one full transmission can be divided into two time slots as shown in Figure 3.

At the first time slot, complex baseband received signal at $\mathbb{D}$ and $\mathbb{R}$ is given by

$$
\begin{aligned}
& \mathbf{y}_{D, 1}=\mathbf{H}_{S D} \mathbf{x}+\mathbf{z}_{D, 1}, \\
& \mathbf{y}_{R, 1}=\mathbf{H}_{S R} \mathbf{x}+\mathbf{z}_{R, 1},
\end{aligned}
$$

where $\mathbf{H}_{S D}$ and $\mathbf{H}_{S R}$ denote two complex circulant channel matrices with their first columns $\left[\mathbf{h}_{S D}^{T}, \mathbf{0}_{1 \times(N-L)}\right]^{T}$ and $\left[\mathbf{h}_{S R}^{T}, \mathbf{0}_{1 \times(N-L / 2)}\right]^{T}$ respectively [20]; $\mathbf{z}_{D, 1}$ and $\mathbf{z}_{R, 1}$ are a realization of a complex additive Gaussian white noise vector with zero mean and covariance matrix $\mathbb{E}\left[\mathbf{z}_{D, 1} \mathbf{z}_{D, 1}^{H}\right]=\mathbb{E}\left[\mathbf{z}_{R, 1} \mathbf{z}_{R, 1}^{H}\right]=$ $\sigma_{n}^{2} \mathbf{I}_{N}$, and $\mathbf{I}_{N}$ is the $N \times N$ identity matrix. Then the relay $\mathbb{R}$ amplifies the received signal $\mathbf{y}_{R, 1}$ and retransmits the signal during the second time slot. The received signal vector at the destination $\mathbb{D}$ is given by

$$
\mathbf{y}_{D, 2}=\beta \mathbf{H}_{R D} \mathbf{H}_{S R} \mathbf{x}+\mathbf{z}_{D, 2},
$$

where $\mathbf{H}_{R D}$ is a circulant channel matrix with first column $\left[\mathbf{h}_{R D}^{T}, \mathbf{0}_{1 \times(N-L / 2)}\right]^{T} ; \mathbf{z}_{D, 2}=\beta \mathbf{H}_{R D} \mathbf{z}_{R, 1}+\widetilde{\mathbf{z}}_{D, 2}$ is a composite noise with zero mean and covariance matrix $\mathbb{E}\left[\mathbf{z}_{D, 2} \mathbf{z}_{D, 2}^{H}\right]=$ $\left(\beta^{2} \mathbf{H}_{R D} \mathbf{H}_{R D}^{H}+\mathbf{I}_{N}\right) \sigma_{n}^{2}$, where $\widetilde{\mathbf{z}}_{D, 2}$ is a realization of a complex additive Gaussian white noise (AWGN) vector with zero mean and covariance matrix $\mathbb{E}\left[\widetilde{\mathbf{z}}_{D, 2} \widetilde{\mathbf{z}}_{D, 2}^{H}\right]=\sigma_{n}^{2} \mathbf{I}_{N}$. Considering long-time averaging, the amplification factor $\beta$ is given by

$$
\beta=\sqrt{\frac{P_{R}}{\sigma_{h}^{2} P_{S}+\sigma_{n}^{2}}},
$$

where $P_{R}$ is the transmit power of relay. Using (2), the effective input-output relation in the AF cooperative communication system can be summarized as

$$
\tilde{\mathbf{y}}=\left[\begin{array}{l}
\mathbf{y}_{D, 1} \\
\mathbf{y}_{D, 2}
\end{array}\right]=\left[\begin{array}{ll}
\mathbf{H}_{S D} & \\
& \beta \mathbf{H}_{R D} \mathbf{H}_{S R}
\end{array}\right]\left[\begin{array}{l}
\mathbf{x} \\
\mathbf{x}
\end{array}\right]+\left[\begin{array}{l}
\mathbf{z}_{D, 1} \\
\mathbf{z}_{D, 2}
\end{array}\right] .
$$

According to the matrix theory [21], all circulant matrices can share the same eigenvectors [20]. That is to say, the same unitary matrix can work for all circulant matrices. Hence, the matrices $\mathbf{H}_{S D}$ and $\mathbf{H}_{R D} \mathbf{H}_{S R}$ in (5) are decomposited as $\mathbf{H}_{S D}=\mathbf{F}^{H} \mathbf{D}_{S D} \mathbf{F}$ and $\mathbf{H}_{R D} \mathbf{H}_{S R}=\mathbf{F}^{H} \mathbf{D}_{S R D} \mathbf{F}$, respectively, where $\mathbf{D}_{S R D}=\mathbf{D}_{R D} \mathbf{D}_{S R}$ denotes a diagonal matrix and $\mathbf{F}$ is the unitary discrete Fourier transform (DFT) matrix with entries $f_{m n}=[\mathbf{F}]_{m n}=1 / \sqrt{N} e^{-j 2 \pi(m-1)(n-1) / N}, m, n=$ $1,2, \ldots, N$. At the same time, $\mathbf{F}^{H} \mathbf{D}_{S R D} \mathbf{F}$ is the decomposition of a circulant matrix which is constructed from a cascaded channel impulse response $\mathbf{h}_{S R D} \triangleq \mathbf{h}_{R D} * \mathbf{h}_{S R}$. Here, both $\mathbf{D}_{S D}$ and $\mathbf{D}_{S R D}$ are diagonal matrices which are given by

$$
\begin{aligned}
\mathbf{D}_{S D} & =\operatorname{diag}\left\{H_{S D}(0), \ldots, H_{S D}(n), \ldots, H_{S D}(N-1)\right\}, \\
\mathbf{D}_{S R D} & =\operatorname{diag}\left\{H_{S R D}(0), \ldots, H_{S R D}(n), \ldots, H_{S R D}(N-1)\right\},
\end{aligned}
$$

respectively, where $H_{S D}(n)$ and $H_{S R D}(n)$ are given by

$$
\begin{aligned}
H_{S D}(n) & =\sum_{l=0}^{L-1} h_{S D}(l) e^{-j 2 \pi n l / N}, \\
H_{S R D}(n) & =\sum_{l=0}^{L-2} h_{S R D}(l) e^{-j 2 \pi n l / N},
\end{aligned}
$$

respectively, where $\mathbf{h}_{S D}=\left[h_{S D}(0), h_{S D}(1), \ldots, h_{S D}(L-1)\right]^{T}$ denotes direct link from source $\mathbb{S}$ to destination $\mathbb{D}$ at the first time slot and $\mathbf{h}_{S R D}=\left[h_{S R D}(0), h_{S R D}(1), \ldots, h_{S R D}(L-2)\right]^{T}$ represents cascaded channel from source $\mathbb{S}$ to destination $\mathbb{D}$ via help of the relay $\mathbb{R}$ at the second time slot. Based on the above analysis, (5) can be rewritten as

$$
\mathbf{y}=\mathbf{X h}+\mathbf{z}
$$

where $\mathbf{y}=\left[\left(\mathbf{F y}_{D, 1}\right)^{T},\left(\mathbf{F y}_{D, 2}\right)^{T}\right]^{T}$ denotes $2 N$-length received signal vector; $\mathbf{X}$ denotes equivalent training matrix, and it can be written as

$$
\mathbf{X}=\left[\begin{array}{cc}
\mathbf{F} \operatorname{diag}(\mathbf{x}) \mathbf{F}_{S D} & \mathbf{0}_{N \times(L-1)} \\
\mathbf{0}_{N \times L} & \mathbf{F} \operatorname{diag}(\mathbf{x}) \mathbf{F}_{S R D}
\end{array}\right],
$$


with $2 N \times(2 L-1)$ dimension; $\mathbf{h}=\left[\begin{array}{ll}\mathbf{h}_{S D}^{T} & \mathbf{h}_{S R D}^{T}\end{array}\right]^{T}$ represents $(2 L-1)$-length cooperative channel vector; $\mathbf{z}=$

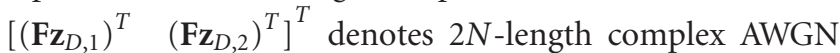
vector; $\mathbf{F}_{S D}$ and $\mathrm{F}_{S R D}$ are partial DFT matrices taking the first $L$ and $(L-1)$ columns of $\mathbf{F}$, respectively. And the $\mathbf{z}$ is a realization of a complex Gaussian random vector with zero mean and covariance matrix $\mathbb{E}\left[\mathbf{z z}^{H}\right]=\left(\beta^{2}\left|\mathbf{D}_{S R}\right|^{2}+\mathbf{I}_{2 N}\right) \sigma_{n}^{2}$.

\section{Sparse Channel Estimation}

In this section, we discuss the sparse channel estimation for AF CCS. Firstly, we review briefly CS theory and restricted isometry property (RIP) of training signal matrix. Then, sparse channel estimation method [17] is introduced. Finally, we propose improved sparse channel estimators by using partial sparse constraint to take full advantage of prior information in AF CCS.

3.1. Review of the CS. In a typical complex sparse identification system, one can use known matrix $\mathbf{U} \in \mathbb{C}^{N \times L}$ to estimate an $L$-length unknown sparse signal vector a based on the observation linear system model:

$$
\mathbf{b}=\mathbf{U} \mathbf{a}+\mathbf{c},
$$

where $\mathbf{b} \in \mathbb{C}^{N}$ is a complex observation signal vector, $\mathbf{c} \in \mathbb{C}^{N}$ is a noise vector, and $\mathbf{a}$ is $K$ sparse vector which means the number of dominant entries is no more than $K$, that is, $\|\mathbf{a}\|_{0} \leq K \ll L$. The position of dominant entries is randomly distributed. In addition, $L \gg N$ according to CS assumption. The optimal sparse solution $\mathbf{a}_{\mathrm{opt}}$ can be obtained uniquely by solving minimization problem:

$$
\mathbf{a}_{\mathrm{opt}}=\arg \min _{\mathbf{a}}\left\{\frac{1}{2}\|\mathbf{b}-\mathbf{U a}\|_{2}^{2}+\lambda_{0}\|\mathbf{a}\|_{0}\right\},
$$

where $\lambda_{0}$ is regularized parameter which trades off the mean square error (MSE) and sparsity. However, solving $\ell_{0}$ norm is NP hard and cannot be utilized in practical applications [22].

Fortunately, alternative suboptimal sparse recovery methods have been studied if the known measurement matrix $\mathbf{U}$ satisfies RIP [23]. Let $\mathbf{U}_{\Omega}, \Omega \subset\{1,2, \ldots, N\}$ be the $N \times|\Omega|$ submatrix extracting those columns of $\mathbf{U}$ that are indexed by the elements of $\Omega$. Then the $K$-restricted isometry constant (RIC) of $\mathbf{U}$ is defined as the smallest parameter $\delta_{K} \in(0,1)$ such that

$$
\left|\frac{\left\|\mathbf{U}_{\Omega} \mathbf{a}_{\Omega}\right\|_{2}^{2}-\left\|\mathbf{a}_{\Omega}\right\|_{2}^{2}}{\left\|\mathbf{a}_{\Omega}\right\|_{2}^{2}}\right| \leq \delta_{K}
$$

for all $\Omega$ with $|\Omega| \leq K$ and all vector $\mathbf{a}_{\Omega} \in \mathbb{C}^{|\Omega|}$. Assume that $\mathbf{U}$ is an $N \times L$ random measurement matrix that satisfies the RIP of order $K$ with RIC $\delta_{K}$, that is, $\mathrm{U} \in \operatorname{RIP}\left(K, \delta_{K}\right)$. Consider an arbitrary sparse vector $\mathbf{a}$ in observation model $\mathbf{b}=\mathbf{U a}+\mathbf{c}$, where $\|\mathbf{c}\|_{2} \leq \xi$, by solving $\ell_{1}$ minimization problem, and suboptimal sparse solution $\hat{\mathbf{a}}_{\text {sub }}$ is obtained by

$$
\hat{\mathbf{a}}_{\mathrm{sub}}=\arg \min _{\mathbf{a}}\left\{\frac{1}{2}\|\mathbf{b}-\mathbf{U a}\|_{2}^{2}+\lambda_{\text {sub }}\|\mathbf{a}\|_{1}\right\},
$$

where $\lambda_{\text {sub }}=C_{0} \cdot \sigma_{n} \log N$ and $C_{0}$ is a parameter which is decided by the noise level and RIC of U. Hence, the estimator $\hat{\mathbf{a}}_{\text {sub }}$ satisfies sparse recovery performance with

$$
\left\|\hat{\mathbf{a}}_{\text {sub }}-\mathbf{a}\right\|_{2} \leq C_{1} \max \left\{\xi, \frac{1}{\sqrt{K}}\left\|\mathbf{a}-\mathbf{a}_{K}\right\|_{1}\right\},
$$

where $C_{1}$ is a parameter which is also decided by noise level and RIC of $\mathbf{U}$. Let us recall the channel estimation problem for AF cooperative systems in (8); if the equivalent training matrix $\mathbf{X}$ satisfies RIP, then accurate sparse channel estimation can be achieved. In the next, we will present improved sparse channel estimation methods by using LASSO algorithm [19].

3.2. Sparse Channel Estimation. Channel estimation is done on sparse channel $\mathbf{h}$ by sending the training symbols. Conventional sparse channel estimation method using LASSO algorithm (SEL) has been proposed for deriving sparse impulse response for AF CCS [17]. According to the system model in (8), the global sparse channel estimator $\widehat{\mathbf{h}}_{\mathrm{SEL}}$ can be achieved by

$$
\widehat{\mathbf{h}}_{\mathrm{SEL}}=\arg \min _{\mathbf{h}}\left\{\frac{1}{2}\|\mathbf{y}-\mathbf{X h}\|_{2}^{2}+\lambda_{\mathrm{SEL}}\left\|\mathbf{W}_{\mathrm{SEL}} \mathbf{h}\right\|_{1}\right\},
$$

where

$$
\mathbf{W}_{\mathrm{SEL}}=\mathbf{I}_{(2 L-1) \times(2 L-1)},
$$

is an identity matrix and $\lambda_{\mathrm{SEL}}=0.02 \sigma_{n} \sqrt{N}$ is a regularization parameter which controls the tradeoff between square error $\|\mathbf{y}-\mathbf{X h}\|_{2}^{2}$ and sparse constrained $\left\|\mathbf{W}_{\mathrm{SEL}} \mathbf{h}\right\|_{1}$. However, the proposed method can only solve global sparse solution well while neglecting the inherent partial sparse structure. In the next, we propose a method to fully exploit the prior information in AF CCS.

3.3. Partial Sparse Channel Estimation. From signal processing perspective, extra prior information of partial sparse can be further utilized. In this situation, partial sparse channel estimation by using LASSO (PEL) $\hat{\mathbf{h}}_{\text {PEL }}$ could be achieved by

$$
\hat{\mathbf{h}}_{\mathrm{PEL}}=\arg \min _{\mathbf{h}}\left\{\frac{1}{2}\|\mathbf{y}-\mathbf{X h}\|_{2}^{2}+\lambda_{\mathrm{PEL}}\left\|\mathbf{W}_{\mathrm{PEL}} \mathbf{h}\right\|_{1}\right\},
$$

where

$$
\mathbf{W}_{\mathrm{PEL}}=\left[\begin{array}{cc}
\mathbf{I}_{L \times L} & \mathbf{0}_{L \times(L-1)} \\
\mathbf{0}_{(L-1) \times L} & \mathbf{0}_{(L-1) \times(L-1)}
\end{array}\right]
$$

is a diagonal weighted matrix and $\lambda_{\mathrm{PEL}}=0.2 \sigma_{n} \sqrt{N}$ is a regularization parameter which controls the tradeoff between square error $\|\mathbf{y}-\mathbf{X h}\|_{2}^{2}$ and local partial sparse constrained $\left\|\mathbf{W}_{\mathrm{PEL}} \mathbf{h}\right\|_{1}$.

Based on the partial sparse constraint on cooperative channel impulse response, we propose an improved PEL (IEL) estimator. On the one hand, the local sparse constrain can improve estimation performance. On the other hand, the global sparse constraint can mitigate noise interference in 


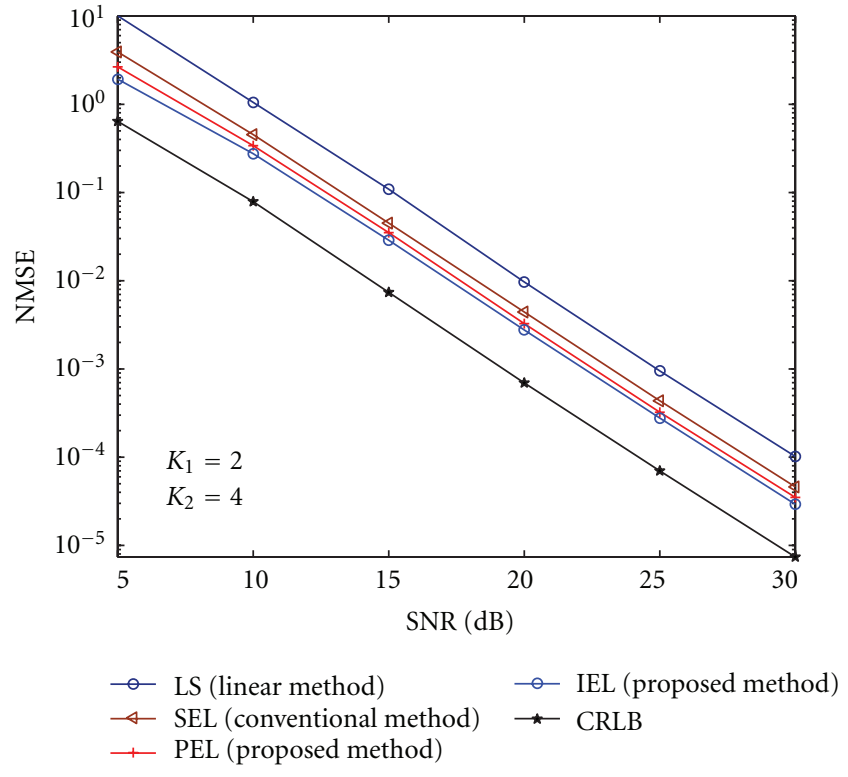

FIGURE 4: Channel estimation performance versus SNR.

the low SNR regime. The IEL estimator $\widehat{\mathbf{h}}_{\text {IEL }}$ can be obtained by

$$
\begin{gathered}
\hat{\mathbf{h}}_{\mathrm{IEL}}=\arg \min _{\mathbf{h}}\left\{\frac{1}{2}\|\mathbf{y}-\mathbf{X h}\|_{2}^{2}+\lambda_{\mathrm{SEL}}\left\|\mathbf{W}_{\mathrm{SEL}} \mathbf{h}\right\|_{1}\right. \\
\left.+\lambda_{\mathrm{PEL}}\left\|\mathbf{W}_{\mathrm{PEL}} \mathbf{h}\right\|_{1}\right\},
\end{gathered}
$$

where the regularization parameters $\lambda_{\mathrm{SEL}}$ and $\lambda_{\mathrm{PEL}}$ are given by the (15) and (18), respectively. In the following, we will give representative simulation results to confirm the effectiveness of the improved sparse channel estimation methods.

\section{Numerical Simulations}

In this section, we will compare the performance of the proposed estimators, that is, PEL and IEL, with SEL estimator and LS estimator. To achieve average estimation performance, 1000 independent Monte-Carlo runs are adopted. The length of training sequence is $N=36$. The length of direct link $\mathbf{h}_{S D}$ is $L=32$ with the number of dominant channel taps $K_{1}=2,4,8$. The two cooperative links $\mathbf{h}_{S R}$ and $\mathbf{h}_{R D}$ have fixed length of $L / 2$ with the number of dominant channel taps $K_{2}=4,8,16$. All of the nonzero channel taps are generated following Rayleigh distribution and set to $\mathbb{E}\left[\left\|\mathbf{h}_{S R}\right\|_{2}\right]=\mathbb{E}\left[\left\|\mathbf{h}_{R D}\right\|_{2}\right]=\mathbb{E}\left[\left\|\mathbf{h}_{S D}\right\|_{2}\right]=1$. Transmit power and AF relay power are fixed as $P_{S}=P_{R}=N P$, where $P$ is the symbol power. The received SNR is defined as $P_{S} / \sigma_{n}^{2}$.

Channel estimator $\hat{\mathbf{h}}$ is evaluated by normalized mean square error (NMSE) which is defined by

$$
\operatorname{NMSE}(\hat{\mathbf{h}})=\frac{\mathbb{E}\left[\|\mathbf{h}-\hat{\mathbf{h}}\|_{2}^{2}\right]}{\|\mathbf{h}\|_{2}},
$$

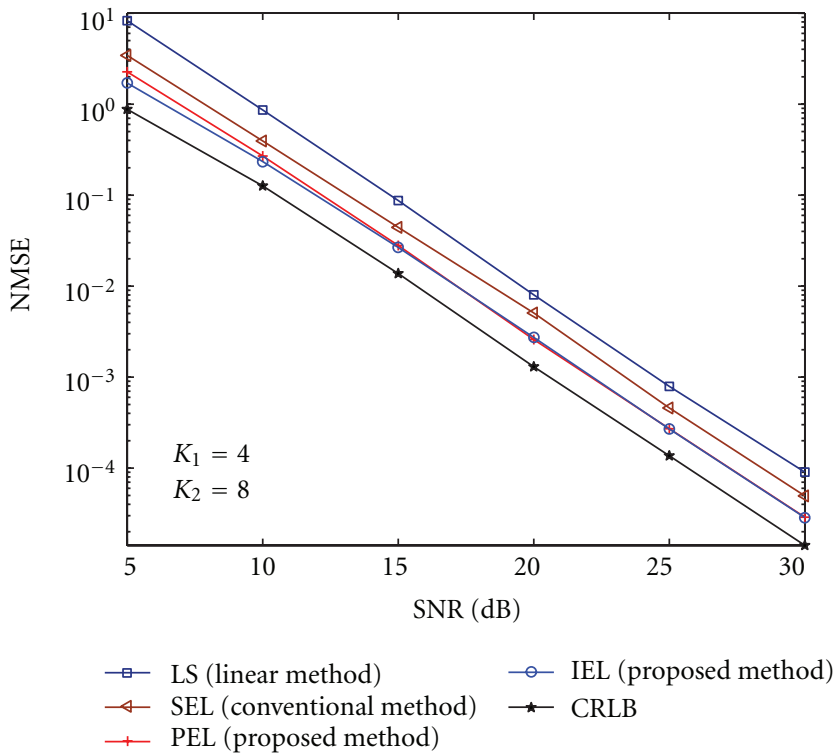

FIGURE 5: Channel estimation performance versus SNR.

where $\mathbf{h}$ and $\hat{\mathbf{h}}$ denote cooperative channel vector and its estimator, respectively. At first, we compare their estimation performance with different number of dominant channel taps $K_{1}$ and $K_{2}$. As shown in Figure 4 , when $K_{1}=2$ and $K_{2}=$ 4, the direct link $\mathbf{h}_{S D}$ is sparse channel impulse response, and the cascaded link $\mathbf{h}_{S R D}$ may not be sparse since the linear convolution between $\mathbf{h}_{S R}$ and $\mathbf{h}_{R D}$. It can be observed from Figure 4 that the two proposed channel estimators are better than both SEL estimator and LS-based linear channel estimator. It is worth nothing that the IEL estimator has a better performance than PEL one, since the IEL takes not only advantage of partial sparse prior information but also utilizes global sparse constraint to mitigate noise interference. The same performance advantage can also be seen in other scenarios with different number of dominant channel taps as shown in Figures 5 and 6 . When $K_{1}=4$ and $K_{2}=8$, we can also find that IEL estimator has a better performance than PEL under low SNR (less than $15 \mathrm{~dB}$ ). On the other hand, if the direct link $\mathbf{h}_{S D}$ is highly sparse, for example, $K_{1}=2$, while the cooperative links $\mathbf{h}_{S R}$ and $\mathbf{h}_{R D}$ are highly dense, for example, $K_{2}=16$, the two proposed channel estimators have a more significant performance advantage over traditional methods. In addition, IEL estimator is worse than PEL estimator when the SNR is higher than $15 \mathrm{~dB}$. According to these results, we can conclude the following: if direct and cooperative links are highly sparse channel, then IEL can achieve obvious better estimation performance than PEL; if the direct link is highly sparse channel while cooperative link is highly dense channel, the estimation performance of the two proposed methods is very close. It is worth mentioning that estimation performance of IEL is better than PEL due to the fact that IEL utilizes global sparse constraint to mitigate noise interference and partial sparse constraint to take advantage of channel sparsity. However, the computational complexity of IEL is higher than PEL. That is to say, IEL uses higher computational complexity than PEL to obtain performance advantage. Hence, to use 


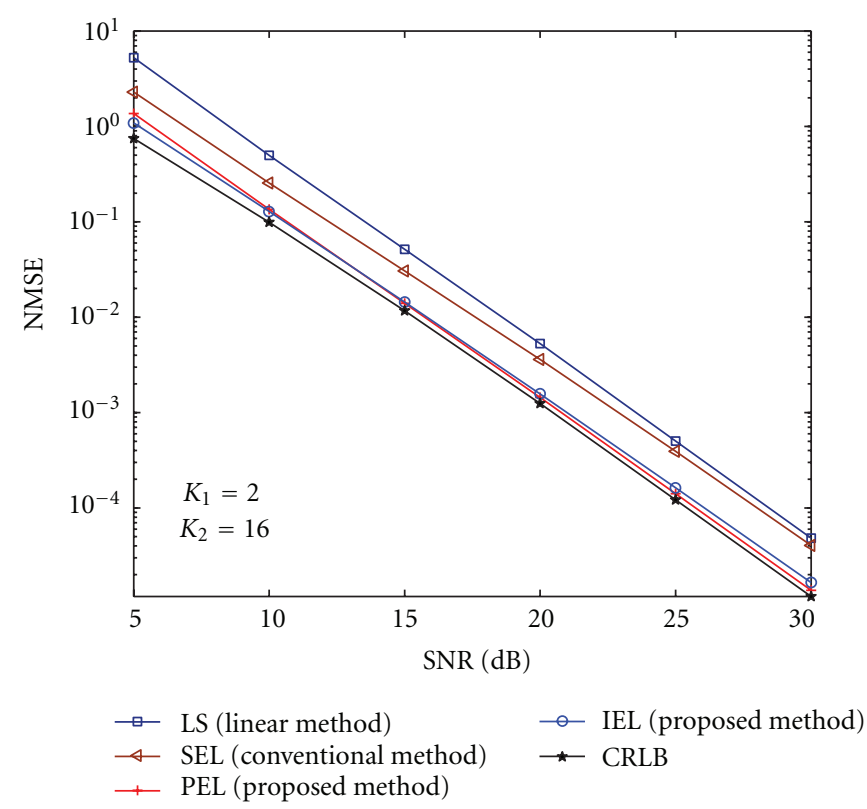

FIGURE 6: Channel estimation performance versus SNR.

IEL or PEL will be decided by the requirement of practical communication systems. In addition, when the number of dominant channel taps is very small, for example, $K_{1}=2$ and $K_{2}=4$, the estimation performance is close to the SEL estimator. However, if the direct link is sparse, for example, $K_{1}=2$ and cooperative link is highly dense, for example, $K_{2}=16$, then the two proposed channel estimators are close to CRLB. According to the previous analysis, we can find that the proposed methods are generalized from both LS-based linear estimation method and SEL, since they are either based on dense or sparse channel assumption. Hence, our proposed methods can work well in different channel environments.

\section{Conclusion}

Accurate CSI is indispensable for coherent detection in AF CCS. Traditional channel estimation methods are based on assumptions of either dense channel model or sparse channel model in AF CCS. In this paper, the two kinds of channel models have been generalized as a partial sparse channel model. By means of compressive sensing and partial sparse constraint, we have proposed improved sparse channel estimation methods to fully exploit channel prior information. Numerical simulations have confirmed the performance superiority of the proposed method over the conventional global sparse channel estimation method and traditional linear LS method. The proposed method can also be extended to other cooperative communication systems such as MIMO AF CCS.

\section{Acknowledgment}

The authors would like to thank Professor Guan Yong Liang for helpful discussions and appreciate the constructive comments of the anonymous reviewers.

\section{References}

[1] T. M. Cover and A. A. E. Gamal, "Capacity theorems for the relay channel," IEEE Transactions on Information Theory, vol. 25, no. 5, pp. 572-584, 1979.

[2] J. N. Laneman, D. N. C. Tse, and G. W. Wornell, "Cooperative diversity in wireless networks: efficient protocols and outage behavior," IEEE Transactions on Information Theory, vol. 50, no. 12, pp. 3062-3080, 2004.

[3] S. Yiu, R. Schober, and L. Lampe, "Distributed space-time block coding," IEEE Transactions on Communications, vol. 54, no. 7, pp. 1195-1206, 2006.

[4] F. Gao, T. Cui, and A. Nallanathan, "On channel estimation and optimal training design for amplify and forward relay networks," IEEE Transactions on Wireless Communications, vol. 7, no. 5, pp. 1907-1916, 2008.

[5] W. Zhuang and M. Ismail, "Cooperation in wireless communication networks," IEEE Wireless Communications, vol. 19, no. 2, pp. 10-20, 2012.

[6] S. Chieochan and E. Hossain, "Cooperative relaying in Wi-Fi networks with network coding," IEEE Wireless Communications, Special Issue on User Cooperation in Wireless Networks, vol. 19, no. 2, pp. 57-65, 2012.

[7] H. Chen, P. Deng, Y. Xu, and X. Li, "A novel localization scheme based on RSS data for wireless sensor networks," in Proceedings of the International Conference on Advanced Web and Network Technologies, and Applications (APWeb '06), Lecture Notes in Computer Science, LNCS, 3842/2006, pp. 315-320, 2006.

[8] H. Chen, M. H. T. Martins, P. Huang, H. C. So, and K. Sezaki, "Cooperative node localization for mobile sensor networks," in Proceedings of the 5th International Conference on Embedded and Ubiquitous Computing (EUC'08), pp. 302-308, Shanghai, China, December 2008.

[9] H. Chen, P. Huang, M. Martins, H. C. So, and K. Sezaki, "Novel centroid localization algorithm for three-dimensional wireless sensor networks," in Proceedings of the International 
Conference on Wireless Communications, Networking and Mobile Computing (WiCOM '08), Dalian, China, October 2008.

[10] E. Telatar, "Capacity of multi-antenna Gaussian channels," European Transactions on Telecommunications, vol. 10, no. 6, pp. 585-595, 1999.

[11] A. Goldsmith, S. A. Jafar, N. Jindal, and S. Vishwanath, "Capacity limits of MIMO channels," IEEE Journal on Selected Areas in Communications, vol. 21, no. 5, pp. 684-702, 2003.

[12] V. Tarokh, N. Seshadri, and A. R. Calderbank, "Space-time codes for high data rate wireless communication: performance criterion and code construction," IEEE Transactions on Information Theory, vol. 44, no. 2, pp. 744-765, 1998.

[13] S. M. Alamouti, "A simple transmit diversity technique for wireless communications," IEEE Journal on Selected Areas in Communications, vol. 16, no. 8, pp. 1451-1458, 1998.

[14] G. Gui, Q. Wan, A. M. Huang, and C. G. Jiang, "Partial sparse multi-path channel estimation using $\ell_{1}$-regularized LS algorithm," in Proceedings of the IEEE Region 10 Conference (TENCON '08), Hyderabad, India, November 2008.

[15] W. U. Bajwa, J. Haupt, G. Raz, and R. Nowak, "Compressed channel sensing," in Proceedings of the 42nd Annual Conference on Information Sciences and Systems (CISS '08), pp. 5-10, Princeton, NJ, USA, March 2008.

[16] G. Tauböck and F. Hlawatsch, "A compressed sensing technique for OFDM channel estimation in mobile environments: exploiting channel sparsity for reducing pilots," in Proceedings of the IEEE International Conference on Acoustics, Speech and Signal Processing (ICASSP '08), pp. 2885-2888, Las Vegas, Nev, USA, April 2008.

[17] G. Gui, P. Wei, A. Mehbodniya, and F. Adachi, "Compressed channel estimation for sparse multipath non-orthogonal amplify-and-forward cooperative networks," in Proceedings of the IEEE 75th Vehicular Technology Conference, Spring, Yokohama, Japan, May 2012.

[18] P. Cheng, L. Gui, Y. Rui, Y. Guo, X. Huang, and W. Zhang, "Compressed sensing based channel estimation for two-way relay networks," IEEE Wireless Communications Letters, vol. 1, no. 3, pp. 201-204, 2012.

[19] R. Tibshirani, "Regression shrinkage and selection via the lasso," Journal of the Royal Statistical Society B, vol. 58, no. 1, pp. 267-288, 1996.

[20] T. H. Pham, A. Nallanathan, and G. H. Krishna, "On the design of optimal training sequence for bi-directional relay networks," IEEE Signal Processing Letters, vol. 16, no. 3, pp. 200-203, 2009.

[21] R. M. Gray, "Toeplitz and circulant matrices: a review," Foundations and Trends in Communications and Information Theory, vol. 2, no. 3, pp. 155-239, 2006.

[22] E. J. Candès, J. Romberg, and T. Tao, "Robust uncertainty principles: exact signal reconstruction from highly incomplete frequency information," IEEE Transactions on Information Theory, vol. 52, no. 2, pp. 489-509, 2006.

[23] E. J. Candès, "The restricted isometry property and its implications for compressed sensing," Comptes Rendus Mathematique, vol. 346, no. 9-10, pp. 589-592, 2008. 

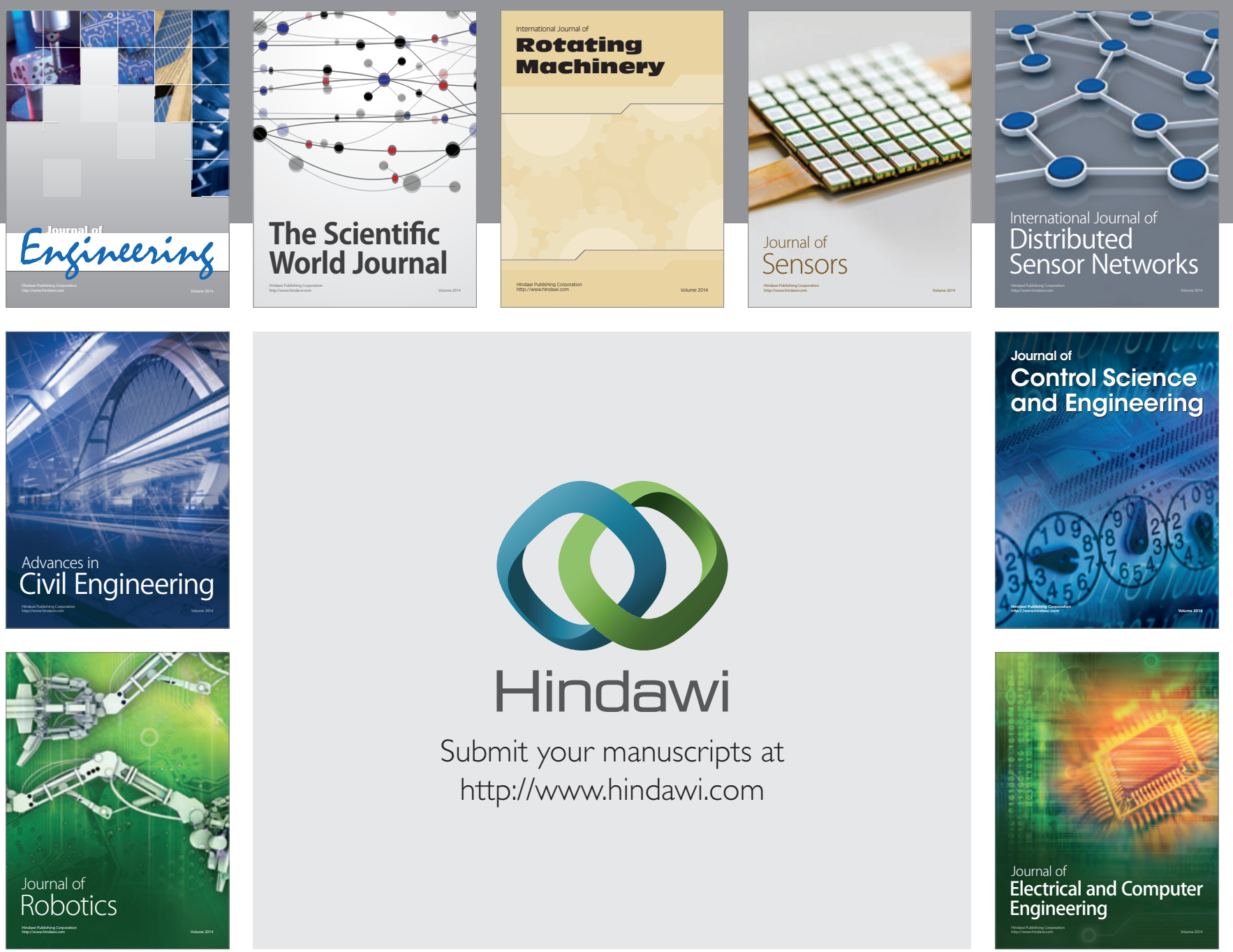

Submit your manuscripts at

http://www.hindawi.com
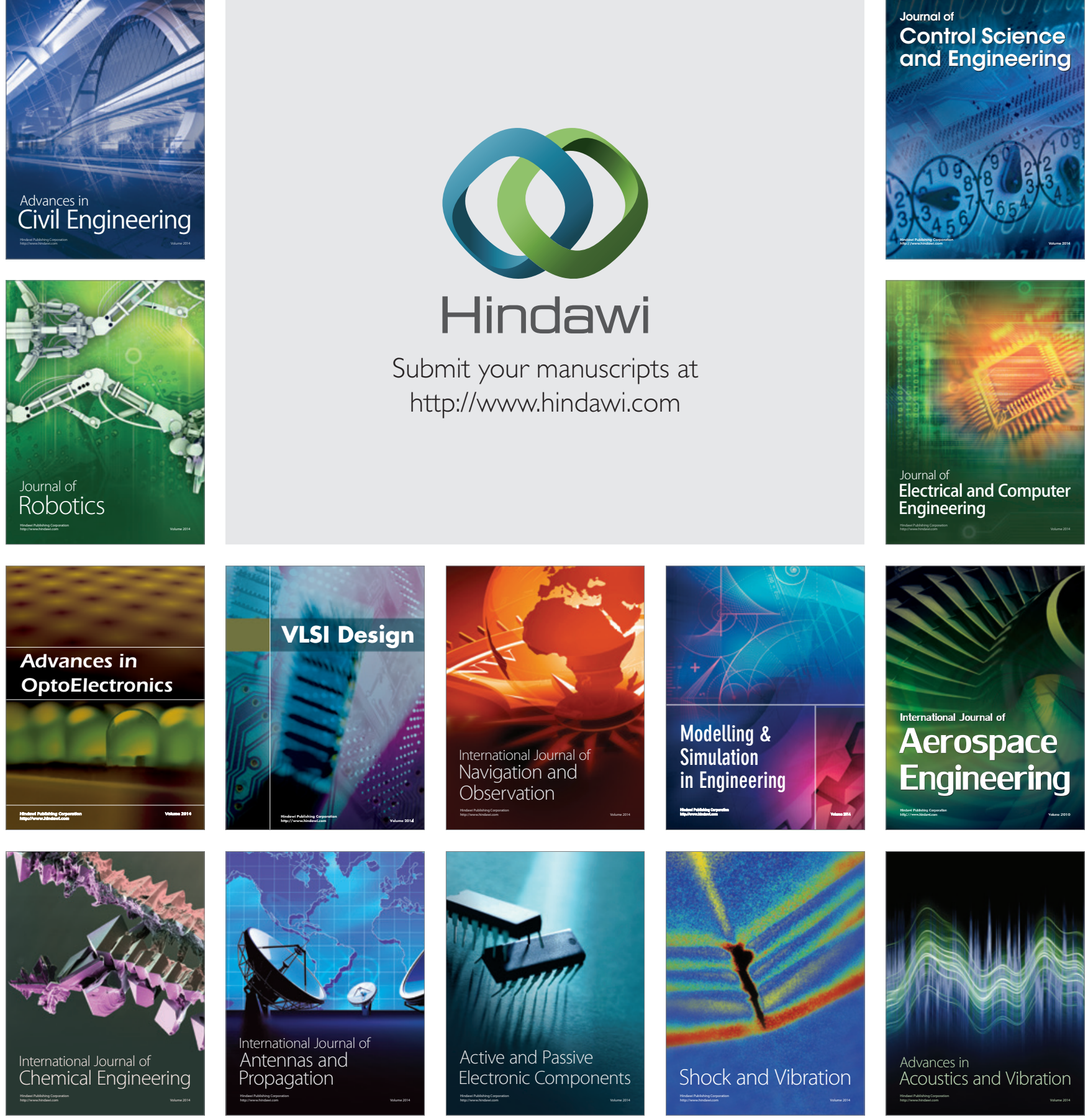